\title{
AUSTRALASIAN JOURNAL OF PARAMEDICINE \\ Review \\ Paramedic health status, fitness and physical tasks: A review of the literature
}

\author{
Samantha Sheridan MSc, FdSc, BSc, is Associate Lecturer $^{1}$
}

Affiliation:

${ }^{1}$ School of Biomedical Sciences, Charles Sturt University, Bathurst, New South Wales

https://doi.org/10.33151/ajp.16.580

\section{Abstract}

\section{Introduction}

Physical fitness and health are closely linked. However, regular workplace assessments of paramedic health and fitness are not conducted, despite increasing evidence that the role requires a high level of fitness due to increasing physical and psychological demands. Describing the physical attributes required to complete the day-to-day tasks of a paramedic is problematic as data has not been collated. Recent registration, and significant illness and injury rates of paramedics in Australia, make systematic identification of the role more important in order to set a standard criteria.

\section{Objective}

This systematic literature review set out to define: 1) paramedic health status and the physical tasks performed; and 2) level of requisite fitness required to complete paramedic tasks.

\section{Results}

Using systematic search strategies, 24 articles were appraised and generally conclude that paramedics have a poorer health status and fitness levels compared to the general population, despite often performing physical heavy handling tasks.

\section{Conclusion}

General themes were highlighted throughout the literature, identifying that further research needs to be conducted to identify the day-to-day tasks of a paramedic and what level of requisite fitness is required. Current health and fitness is lacking for physical heavy handling activities performed in the paramedic role.

\section{Keywords:}

paramedic; emergency medical technician; health; fitness; physical tasks

Corresponding Author: Sam Sheridan, ssheridan@csu.edu.au 


\section{Introduction}

Being physically fit may be defined as the ability to complete daily tasks without excessive fatigue, leaving energy left over for leisure activities and unforeseen emergencies (1). Components measured that contribute to fitness include those related to health such as muscular strength, muscular endurance, body composition and flexibility, and those that relate to athletic skill. These components are often used to measure health status, ie. the current health level and ability to complete certain tasks (2). Therefore to understand if paramedics are physically fit it is important to understand the daily tasks performed and their health status. The World Health Organization (3) defines health as 'a state that incorporates physical, mental and social wellbeing', therefore it is safe to assume that there is a significant relationship between health, health status and physical fitness.

There is a prevalence of what might be considered 'poor health' in paramedics including high stress, depression, cardiovascular problems $(4,5)$ and obesity $(6)$, which potentially impacts on their ability to cope with the physical and other aspects of this demanding role. Generally, paramedics can have a negative perception or attitude toward their own health and fitness, perhaps due to factors such as shift-work $(7,8)$. Assessing health and fitness is problematic because of perceived negativity linked to fear of job loss (9). However, evidence from research in Ireland and the Czech Republic indicates paramedics require a certain level of fitness, and therefore health status, due to significant increases in physical and psychological demands of the role $(10,11)$. Overall, a lack of fitness may contribute to poor paramedic health and risks such as cardiovascular disease (CVD) (10-12). The physical aspects of the paramedic role and what tasks constitute the day-to-day job, are still largely unquantified. Studies specifically on emergency ambulance work have mostly focussed on the prevalence of the relationship between post-traumatic stress and exposure to distressing events (13). Research which describes the physical attributes of emergency medical services and paramedics is lacking, unable to define the level of requisite fitness, despite the challenging physical, psychological working environment and job requirements (14).

What is evident is that paramedic services may have an 'onboarding' or fitness assessment for new recruits to assess their ability to do the work as described in the service's job description. However, on-boarding, pre-employment healthrelated fitness tests (HRFT), physical aptitude tests and/or workers health surveillance activities $(4,9)$ are not standardised across ambulance services. This process is unregulated, with no clear guidance or legislation regarding specific requirements, and wholly dependent on the ambulance service employer. Once employed, health and fitness assessments to perform the job are generally not continued through a paramedic's career (9). Paramedics perform sporadic high physical exertion tasks, such as cardiopulmonary resuscitation
(CPR) (14), between periods of relatively sedentary activity, which could place paramedics at high risk of CVD $(6,10,11)$. It is important to monitor and facilitate health and fitness levels to reduce this risk.

Expectations of the paramedic role, including fitness, will become a matter of national focus now that registration has been introduced (in December 2018) (15). While the quantifiable demands of paramedic work is not readily evident, what is evident is the growing amount of research on illness and injury rates (16). This review aims to explore what the health status of paramedics is, what physical tasks are performed, and what fitness levels are required to complete these tasks.

\section{Method}

A comprehensive literature review was conducted using EBSCOhost, ProQuest, Ovid, Scopus, Sage, OpenGrey and Wiley Online Library. Keywords and MeSH terms used (depending on database) were: paramedic, emergency medical technician, EMT, ambulance officer, ambulance attendant, firefighter, fireman, firewoman, police, law enforcement officers, health, fitness, physical fitness, physical capacity, physical condition, perception, attitude, health perceptions, self-concept. Due to the difficulty in finding relevant results, exclusion criteria were kept to a minimum.

Three search strategies were conducted. Located article results, titles and abstracts were reviewed for relevance to the research question. Reference lists were also appraised to complement electronic database searches, providing a total of 24 articles for critical appraisal. After implementation of exclusion criteria, three assessments were made of the identified literature including: the relevance of the article to the research question, whether the literature at the top of the hierarchy of evidence had been identified fully, and the quality of evidence that had been included (17). Relevance of evidence was then established according to what articles or studies would answer the research question (Figure 1).

\section{Results}

The 24 included articles comprised of four literature reviews, 17 quantitative studies and three qualitative and mixed method studies. Nine articles were excluded from the results as they did not specifically address paramedics, however, they are relevant to background and discussion due to significant crossover in attributes and characteristics affecting fitness for the role.

\section{Paramedic health status}

The physical tasks and the demands of the role, such as handling heavy patients and equipment and awkward postures due to the unpredictable environment, are significantly associated with injury (13). 
Aasa and colleagues (13) in cross-sectional study of 1500 ambulance personnel determined that more than $50 \%$ of reported injuries were due to these activities. This task-injury linkage is further supported by a biomechanical examination of task simulations undertaken by Lavender and colleagues (18). This study showed (using a logistic regression model) that even when handling a mannequin weighing only $48 \mathrm{~kg}$ (in a simulation task), paramedics complete daily hazardous tasks associated with lifting and awkward positions (18).

Six cross-sectional, international studies identify that excess weight is common in paramedics $(6,8,10,11,14,19)$ lowering quality of work and therefore the ability to perform physical activities. In a sample of paramedics assessed via survey (8), those with self-reported high body mass index (BMI) assess their health status as worse than those with lower reported $\mathrm{BMI}$, which could result in physical limitations affecting tasks performed. This is supported in a randomised controlled trial (20), comparing experimental $(n=8)$ and control subjects $(n=6)$, concluding that a twice weekly exercise intervention, combating the sedentary nature of the role and difficult activities performed, improves physical fitness therefore improving work capacity and performance (20). The experimental group showed significant improvements in flexibility and maximal oxygen uptake (VO2 max) for example, which are commonly assessed at pre-employment. As well as reporting high BMI, paramedics report $14 \%$ less physical activity than the general public and higher levels of fatigue and stress (5). Bridgewater and colleagues (21) conducted a study investigating whether CPR performance decreased with age, finding no significant difference during 10 minutes of CPR. However, an inadvertent finding was demonstrated indicating the workload achieved is enough to induce a myocardial infarction in those with CVD.

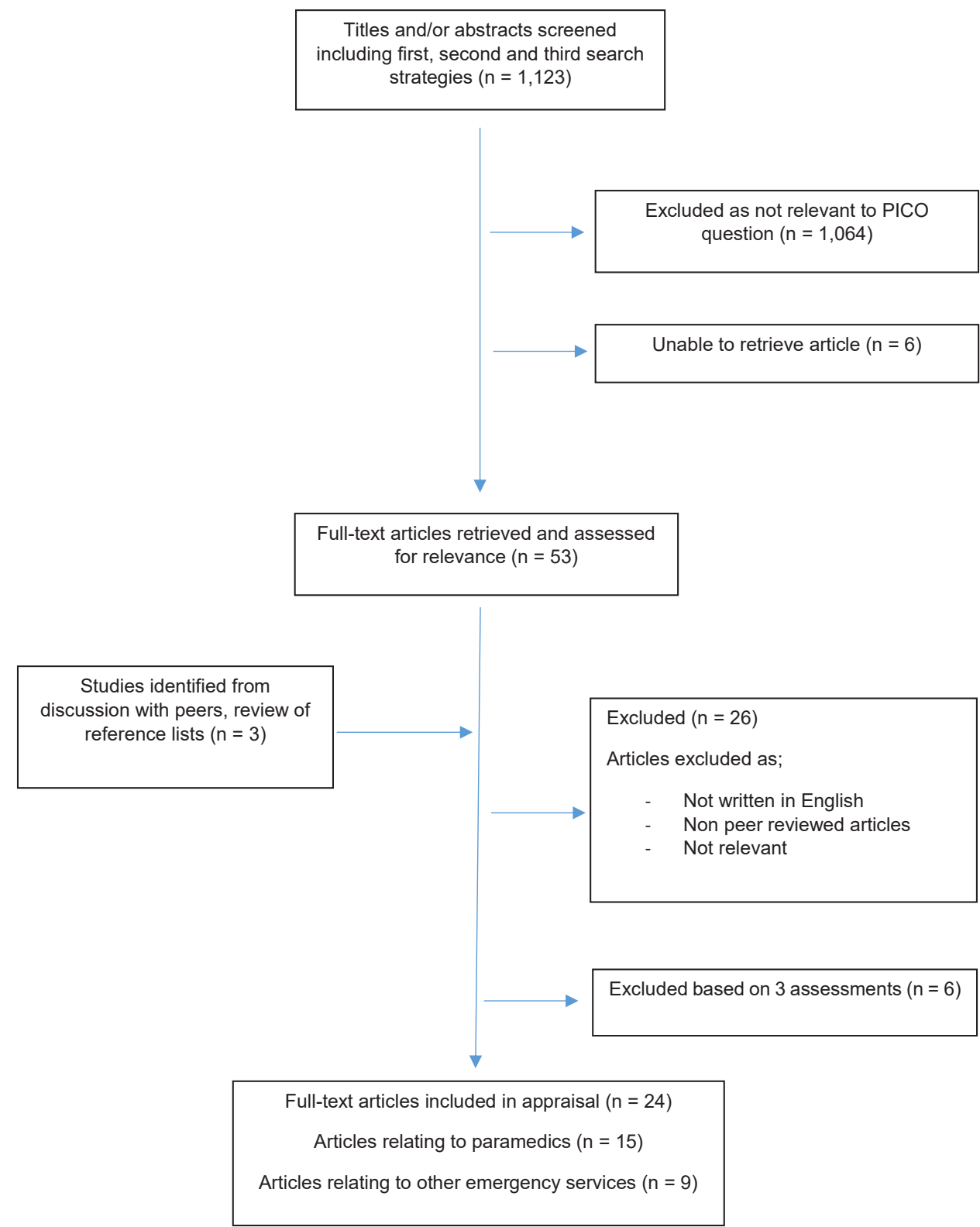

Figure 1. Flowchart of study selection process for inclusion within literature review 
A retrospective analysis of de-identified data from a cohort of Bachelor of Paramedic Science students who participated in a simulated on-boarding/pre-employment test as per the Queensland Ambulance Service (QAS) pre-employment HFRT (4). Participants completed nine component tests forming the HFRT including the Purdue peg-board test, unilateral grip strength, and back strength and leg strength dynamometer tests. Also included were the seven and 60 -stage sit-up tests, the three-minute step test, sit and reach test and the trunk rotational flexibility test (22). Strict scoring and protocols according to QAS HFRT were adhered to throughout the study. The results indicated that the inclusion of tests that do not represent tasks performed by a paramedic mean the preemployment test does not reliably measure recruits' fitness to perform to role (4).

Paramedics perceive physical exercise as important to their role, and necessary to perform tasks, but feel support is lacking in the workplace. Pre-employment tests are completed that do not represent the role, and regular participation and maintenance of physical health in the workplace is a health and safety risk (7).

\section{Fitness levels required for paramedics}

Despite concern regarding CVD risk, obesity prevalence affecting performance and injury risk, identifying what fitness levels should be is not readily apparent. Investigations have used a multitude of tests based on perceptions of the role predominantly based on other emergency services or selfreported by paramedics. Those considered most valid include three separate investigations $(18,21,23)$ which all incorporate simulation or physical activities directly observed as performed in the role, such as lifting or CPR. It has been identified that fitness measures, such as VO2 max, flexibility and strength $(14,23)$ are important to performance of physical activities but have not identified the specific levels or goals (10) and there is currently no validated model that simulates paramedic work (4). A meta-analysis of 25 articles in 2014 by Hegg-Deloye et al (24) identifies a gap in research in this area. Literature was found specifically identifying firefighters risk to CVD where cross-over analysis between roles are made $(25,26)$, but a paucity of data regarding paramedic specific risk.

A cross-sectional study evaluating the fitness of ambulance personnel in Belfast attempted to quantify the physical demands of accident and emergency work (11) and found more than half of paramedics were overweight. This prompted the investigators to carry out a randomised controlled trial investigating the effect of a 10-week exercise intervention on working capacity and physical fitness compared to no intervention (20). Although BMI was comparable to the general population $(10,11)$, this still placed them at risk of CVD.

Paramedics were also found to have difficulties in completing short periods of anaerobic work (11). The researchers sought to see if an exercise intervention would improve outcomes
(20). The exercise intervention group completed two intensive training sessions per week for 10 weeks in total. Comparisons of changes in physical working capacity were made by measuring heart rate, blood pressure, anaerobic fitness, explosive leg power, lower back and hamstring flexibility and abdominal strength (20). Although valid measures in sport and fitness, the relevance of these measures as well as the activities to the role of paramedics was not discussed by the authors. Improvements were shown in flexibility, sit-up performance, standing broad jump, VO2 max, lactate concentration and anaerobic thresholds.

Physical activities such as CPR are performed below anaerobic capacity (14) and can be performed well by both the layperson and paramedics, however, a certain level of fitness is beneficial to its effectiveness (27). In a non-randomised control study $(n=28)$, sedentary paramedics recorded significantly higher heart rates and VO2 max scores than physically active inexperienced rescuers suggesting a slower recovery (27). A comparative study by Buzga and colleagues (10) carried out in the Czech Republic assessed and compared student paramedics' $(n=32)$ physical fitness to professional paramedics $(n=42)$ finding that the professional paramedics have significantly higher body weights $(p<0.05)$ and $\mathrm{BMI}(\mathrm{p}<0.001)$ but are still comparable to the general population (10). In an American study, a retrospective analysis of emergency responders (6) reviewed consecutive pre-placement medical examinations of firefighters and ambulance recruits between 2004 and 2007 ( $n=370)$. This study investigated the prevalence of excess weight among recruits and any physiological associations with BMI to validate its use as a measure for excess adiposity in recruits (6). Overall, paramedics were found to have proportional body weights and BMls when compared to the general public (10). Obesity is not only linked to CVD but affects task completion as increased body fat positively correlates with demand placed on the body (14). This is clear when minimising risk of injury when performing heavy handling and awkward postures.

\section{Discussion}

Although an identified theme, there is inconsistency in the literature when identifying what level of fitness a paramedic needs to attain, however, the implications of obesity and CVD in this population are clear. Although there may be no benefit from high fitness levels for CPR performance $(21,27)$ there is a benefit for carrying out physical activities in the role such as patient handling and mitigating awkward postures (18), improving performance and reducing injury rates. The challenge of reducing those risks and achieving requisite fitness are complex.

Physical fitness is suggested as being poor among paramedics due to the prevalence of obesity $(6,10,28)$. Similar to firefighters, paramedics are at risk of CVD $(11,14,28,29)$. 
Due to the physical tasks performed in the role, a requisite health status and fitness is required (6), and although this is assessed at the pre-employment level, it is not monitored regularly (8) and therefore allowed to decline (as suggested by injury rates) (16). This should be considered a safety issue for paramedics and the patients they treat. Gamble and colleagues (20) suggested that a twice weekly exercise intervention improved physical fitness, increased work capacity and would decrease absenteeism (20). This study involved a small sample, which may have been subject to selection bias but is consistent with other studies (14), identifying that the relevance of the exercise measurements and interventions to the role of a paramedic should be established.

It is evident in the literature that excess weight is prevalent in paramedics and is comparable to the general population (10). It is associated with elevated cardiovascular risk among future emergency responders or paramedics $(6,10)$. Although it should be noted that the data used in studies such as by Buzga, Jirak and Buzgova (10) to compare to the general population were significantly out-of-date and not representative of the population's actual current levels, it is still thought that physical fitness is associated with injury risk and ability to perform tasks. Fitness levels are often measured using progressive incremental stress tests performed on a bicycle ergometer for example $(10,14,23)$, which although a recognised method to measure fitness, are not tasks specifically performed by paramedics. Similar to HFRT (4) therefore, the ideal fitness level to undertake the role of paramedic effectively cannot be identified presently.

It has been concluded that firefighters are at increased risk of CVD due to job-specific demands, such as carbon monoxide and fume exposure, but also because of shift-work, poor dietary habits, high cholesterol levels and obesity $(19,28)$. Some of these factors will also contribute to the prevalence of obesity in paramedics (6), such as shift patterns and dietary habits on shift. Fitness interventions should therefore be culturally relevant to the ethos and perceptions of those at whom it is targeted (30) as well as being task specific. Not surprisingly, most paramedics begin their working life 'fit'. Recruits who are motivated to maintain physical fitness for pre-employment testing reduce the use of training programs once employed (31), therefore losing fitness. Paramedic fitness decreases and body weight increases once a person is employed, suggesting an increased susceptibility to CVD risk factors (10). This is perhaps due to time constraints presented by shift-work and the culture of the organisation. The QAS expects employees to maintain fitness but does not provide organisational support to facilitate it (32), such as time on station or equipment to exercise. It may be perceived that once employed health and fitness does not matter, particularly if the on-boarding assessment tool is unvalidated (4). However, more care should be taken to maintain health and fitness levels to reduce the risk of CVD and injury rates, and the ability to perform necessary tasks.

Three studies suggest excess weight and risk factors for CVD, such as high blood pressure and poor diet, are prevalent in paramedics or emergency service workers $(6,11,14)$, but may still be comparable to the general population (10). It is also suggested that further investigation is necessary (6). There is no apparent minimum standard fitness level for a paramedic. It may reasonably be assumed that a paramedic should be healthy. Throughout the literature a paucity in data is apparent (33), it is therefore recommended by this author that a longitudinal study identifying physical tasks performed and the requisite fitness levels to perform them would be beneficial.

Based on the literature it is interesting to see that the paramedic role and appropriate assessment tasks or criteria have not yet been validated. Therefore, current pre-employment HFRT may not be considered as accurate indicators of a recruit's ability to complete the role (4). It is difficult to define a paramedic, there is an absence of research in this area, and ergonomics of the role have not been examined, therefore further research investigating what the occupational demands and physical standards are for paramedics may be pertinent, before re-designing HRFT making it a more suitable pre-employment fitness screening tool (4). Perhaps a comparative study of numerous HRFTs between different ambulance services, with exclusion rates, injury rates and prevalence of obesity may indicate what a more reliable HRFT may look like.

Overall, paramedics are at risk of CVD and obesity, therefore establishing and maintaining regular physical activity, monitoring and support is vital. The literature is consistently lacking in what is deemed an appropriate fitness level for a paramedic and how best to assess and monitor this. It is therefore recommended that further research be conducted. Before an ideal physical fitness level is identified the role has to be established then physical fitness recommendations can be made. Assessments of which may be validated and standardised, consisting of testing modalities relevant to everyday tasks carried out by paramedics.

\section{Limitations}

Limitations to the search strategies must be noted; some articles appeared pertinent to the research proposal yet the author was unable to access them through university databases. It is also acknowledged the search strategy may show discrepancies from database-to-database because different formats and use of coding was required. Also, limited resources were available and so the search and appraisal of the literature was conducted by the author only, therefore it is possible some references may have been missed. 


\section{Conclusion}

A systematic literature review was conducted to define the routine physical activities performed by paramedics, and fitness levels required to complete these tasks. It was found that overall, paramedics are at risk of CVD, obesity and injury $(6,10,11)$. Studies into perceptions of paramedics, firefighters and police officers suggest physical activity is considered important $(7,34,35)$ but not routinely monitored with no set level or standard recommended. It is thought that testing and maintenance of physical activity is important to reduce workrelated injury (29), decrease cardiovascular risk and obesity, and improve ability to perform tasks. There is a paucity of data despite the relevance and importance of the area to paramedicine.

There is general consensus that the physical demands of the role require or will benefit from maintained fitness levels. However, there does not seem to be consensus regarding what the ideal levels is. Therefore, further investigation needs to be conducted to identify the day-to-day tasks of the paramedic to be able to implement appropriate workplace assessments, and to improve performance with maintained appropriate levels of health and fitness.

\section{Conflict of interest}

The author of this paper reports no competing interests and has completed the ICMJE conflict of interest statement.

\section{Acknowledgements}

The author thanks Alexander (Sandy) MacQuarrie for his mentorship, guidance and support.

\section{References}

1. Caspersen C, Powell K, Christenson G. Physical activity, exercise, and physical fitness: definitions and distinctions for health-related research. 1985. Available at: www.ncbi. nlm.nih.gov/pmc/articles/PMC1424733/

2. Bergner M, Rothman M. Health status measures: an overview and guide for selection. Annu Rev Public Health 1987;8:191-210.

3. World Health Organization, editor Preamble to the Constitution of WHO as adopted by the International Health Conference. International Health Conference; 1948; New York. Available at: https://hero.epa.gov/hero/index.cfm/ reference/details/reference_id/80385

4. Thornton K, Sayers M. Unfit for duty? Evaluation of 4 years of paramedic preemployment fitness screening test results. Prehosp Emerg Care 2014;18:201-6.

5. Courtney J, Francis A, Paxton S. Caring for the carers: fatigue, sleep, and mental health in Australian paramedic shiftworkers. The Australian and New Zealand Journal of Organisational Psychology 2010;3:32-41.

6. Tsismenakis A, Christophi C, Burress J. The obesity epidemic and future emergency responders. Obesity (Silver Spring) 2009;17:1648-50.

7. Rice V, Glass N, Ogle K, Parsian N. Exploring physical health perceptions, fatigue and stress among health care professionals. J Multidiscip Healthc 2014;7:155-61.

8. Pek E, Fuge K, Marton J, et al. Cross-sectional survey on self-reported health of ambulance personnel. Scand J Trauma Resusc Emerg Med 2015;23:14.

9. Plat M, Frings-Dresen M, Sluiter J. A systematic review of job-specific workers' health surveillance activities for firefighting, ambulance, police and military personnel. Int Arch Occup Environ Health 2011;84:839-57.

10.Buzga M, Jirak Z, Buzgova R. State of physical health and fitness of paramedics in Czech Republic. Wulfenia 2015;22(3).

11. Gamble R, Stevens A, McBrien H, et al. Physical fitness and occupational demands of the Belfast Ambulance Service. $\mathrm{Br}$ J Ind Med 1991;48:592-6.

12. Bennett P, Williams Y, Page N, Hood K, Woollard M. Levels of mental health problems among UK emergency ambulance workers. Emerg Med J 2004;21:235-6.

13.Aasa U, Barnekow-Bergkvist M, Ängquist K, Brulin C. Relationships between work-related factors and disorders in the neck-shoulder and low-back region among female and male ambulance personnel. J Occup Health 2005;47:481-9.

14.Chapman D, Peiffer J, Abbiss C, Laursen P. A descriptive physical profile of Western Australian male paramedics. Journal of Emergency Primary Health Care (JEPHC) 2007;5:1829-41.

15.Paramedics Australasia. Registration: Paramedics Australasia; 2014. Available at: www.paramedics.org/ advocacy/registration/

16.Maguire BJ, O'Meara PF, Brightwell RF, O'Neill BJ, Fitzgerald GJ. Occupational injury risk among Australian paramedics: an analysis of national data. Med J Aust 2014;200:477-80.

17. Aveyard H. Doing a literature review in health and social care. Second edn: Open University Press; 2010.

18. Lavender S, Conrad K, Reichelt P, Johnson P, Meyer F. Biomechanical analyses of paramedics simulating frequently performed strenuous work tasks. Appl Ergon 2000;31:167-77.

19.Byczek L, Walton SM, Conrad KM, Reichelt PA, Samo DG. Cardiovascular risks in firefighters implications for occupational health nurse practice. AAOHN J 2004;52:6676.

20.Gamble R, Boreham C, Stevens A. Effects of a 10-week exercise intervention programme on exercise and work capacities in Belfast's ambulancemen. Occup Med (Lond) 1993;43:85-9. 


\section{References (continued)}

21. Bridgewater F, Bridgewater K, Zeitz C. Using the ability to perform CPR as a standard of fitness: a consideration of the influence of aging on the physiological responses of a select group of first aiders performing cardiopulmonary resuscitation. Resuscitation 2000;45:97-103.

22. Queensland Ambulance Service. Undergraduate Student University Clinical Placement Kit. In: Service QA, editor: Queensland Government; 2014.

23. Barnekow-Bergkvist M, Aasa U, Angquist KA, Johansson $\mathrm{H}$. Prediction of development of fatigue during a simulated ambulance work task from physical performance tests. Ergonomics 2004;47:1238-50.

24. Hegg-Deloye S, Brassard P, Jauvin N, et al. Current state of knowledge of post-traumatic stress, sleeping problems, obesity and cardiovascular disease in paramedics. Emerg Med J 2014;31:242-7.

25. Banes $C$. Firefighters' cardiovascular risk behaviors effective interventions and cultural congruence. Workplace Health Saf 2014;62:27-34.

26. Drew-Nord D, Hong O, Froelicher E. Cardiovascular risk factors among career firefighters. Continuing Education 2009;57:415-24.

27. Lucia A, de las Heras J, Perez M, et al. The importance of physical fitness in the performance of adequate cardiopulmonary resuscitation. Chest 1999;115:158-64.

28. Baur D, Christophi C, Tsismenakis A, Jahnke S, SN K. Weight- perception in male career firefighters and its association with cardiovascular risk factors. BMC Public Health 2012;12:480-8.

29.Broniecki M, Esterman A, May E, Grantham H. Musculoskeletal disorder prevalence and risk factors in ambulance officers. J Back Musculoskelet Rehabi 2010;23:165-74.

30.Staley JA, Weiner B, Linnana L. Firefighter fitness, coronary heart disease, and sudden cardiac death risk. Am J Health Behav 2011;35:5603-17.

31.Lagestad $P$, van den Tillaar $R$. Longitudinal changes in the physical activity patterns of police officers. International Journal of Police Science \& Management 2014;16:76-86.

32. Queensland Ambulance Service. Role description - advanced care paramedic. Department of Health, Queensland Government; 2015.

33. Hegg-Deloye S, Brassard P, Prairie J, et al. Prevalence of risk factors for cardiovascular disease in paramedics. Int Arch Occup Environ Health 2015;88:973-80.

34.Betlehem J, Horvath A, Jeges S, et al. How healthy are ambulance personnel in Central Europe? Eval Health Prof 2014;37:394-406.

35. Jahnke S, Poston W, Jitnarin N, Haddock K. Health concerns of the U.S. Fire Service: perspectives from the firehouse. Am J Health Promot 2012;27:111-8. 